demonstration using a CCR6 EGFP knock-in mouse. Eur J Immunol 2002; 32: 104-112

22. McDonald KG, McDonough JS, Wang $\mathrm{C}$ et al. CC chemokine receptor 6 expression by B lymphocytes is essential for the development of isolated lymphoid follicles. Am J Pathol 2007; 170: 1229-1240

23. Neusser MA, Kraus AK, Regele H et al. The chemokine receptor CXCR7 is expressed on lymphatic endothelial cells during renal allograft rejection. Kidney Int 2010; 77: 801-808

24. Cohen CD, Frach K, Schlondorff D et al. Quantitative gene expression analysis in renal biopsies: a novel protocol for a high-throughput multicenter application. Kidney Int 2002; 61: 133-140

25. Segerer S, Cui Y, Hudkins KL et al. Expression of the chemokine monocyte chemoattractant protein-1 and its receptor chemokine receptor 2 in human crescentic glomerulonephritis. J Am Soc Nephrol 2000; 11: 2231-2242

26. Ghadjar P, Rubie C, Aebersold DM et al. The chemokine CCL20 and its receptor CCR6 in human malignancy with focus on colorectal cancer. Int J Cancer 2009; 125: 741-745

27. Tanida S, Yoshitomi H, Nishitani K et al. CCL20 produced in the cytokine network of rheumatoid arthritis recruits CCR6+ mononuclear cells and enhances the production of IL-6. Cytokine 2009; 47: 112-118

28. Brand S. Crohn's disease: Th1, Th17 or both? The change of a paradigm: new immunological and genetic insights implicate Th17 cells in the pathogenesis of Crohn's disease. Gut 2009; 58: 1152-1167

29. Williams IR. CCR6 and CCL20: partners in intestinal immunity and lymphorganogenesis. Ann NY Acad Sci 2006; 1072: 52-61

30. Lugering A, Floer M, Westphal S et al. Absence of CCR6 inhibits $\mathrm{CD} 4+$ regulatory T-cell development and $\mathrm{M}$-cell formation inside Peyer's patches. Am J Pathol 2005; 166: 1647-1654

31. Westphal S, Lugering A, von Wedel J et al. Resistance of chemokine receptor 6-deficient mice to Yersinia enterocolitica infection: evidence of defective M-cell formation in vivo. Am J Pathol 2008; 172: 671-680
32. Turner JE, Paust HJ, Steinmetz OM et al. CCR6 recruits regulatory $\mathrm{T}$ cells and Th17 cells to the kidney in glomerulonephritis. J Am Soc Nephrol 21: 974-985

33. Cohen CD, Calvaresi N, Armelloni S et al. CD20-positive infiltrates in human membranous glomerulonephritis. J Nephrol 2005; 18: 328-333

34. Segerer S, Mack M, Regele H et al. Expression of the C-C chemokine receptor 5 in human kidney diseases. Kidney Int 1999; 56: 52-64

35. Woltman AM, de Fijter JW, van der Kooij SW et al. MIP-3alpha/ CCL20 in renal transplantation and its possible involvement as dendritic cell chemoattractant in allograft rejection. Am J Transplant 2005; 5: 2114-2125

36. Gerritsen ME, Tomlinson JE, Zlot C et al. Using gene expression profiling to identify the molecular basis of the synergistic actions of hepatocyte growth factor and vascular endothelial growth factor in human endothelial cells. Br J Pharmacol 2003; 140: 595-610

37. Hillyer P, Mordelet E, Flynn G et al. Chemokines, chemokine receptors and adhesion molecules on different human endothelia: discriminating the tissue-specific functions that affect leucocyte migration. Clin Exp Immunol 2003; 134: 431-441

38. Punj V, Matta H, Schamus S et al. Induction of CCL20 production by Kaposi sarcoma-associated herpesvirus: role of viral FLICE inhibitory protein K13-induced NF-kappaB activation. Blood 2009; 113 : 5660-5668

39. Li M, Ransohoff RM. The roles of chemokine CXCL12 in embryonic and brain tumor angiogenesis. Semin Cancer Biol 2009; 19: 111-115

40. Takabatake Y, Sugiyama T, Kohara H et al. The CXCL12 (SDF-1)/ CXCR4 axis is essential for the development of renal vasculature. $J$ Am Soc Nephrol 2009; 20: 1714-1723

41. Beider K, Abraham M, Begin M et al. Interaction between CXCR4 and CCL20 pathways regulates tumor growth. PLOS ONE 2009; 4: e5125

Received for publication: 20.12.09; Accepted in revised form: 19.8.10

\title{
Anti-C1q autoantibodies do not correlate with the occurrence or severity of experimental lupus nephritis
}

\author{
Cornelia Bigler ${ }^{1}$, Helmut Hopfer ${ }^{2}$, Doris Danner ${ }^{1}$, Monica Schaller ${ }^{1}$, Michael J. Mihatsch ${ }^{2}$ \\ and Marten Trendelenburg ${ }^{1}$
}

${ }^{1}$ Clinical Immunology, Department of Biomedicine, University Hospital Basel, Switzerland and ${ }^{2}$ Institute for Pathology, University Hospital Basel, Switzerland

Correspondence and offprint requests to: Marten Trendelenburg; E-mail: marten.trendelenburg@unibas.ch

\begin{abstract}
Background. In systemic lupus erythematosus patients, a strong association between the occurrence of antibodies against complement $\mathrm{C} 1 \mathrm{q}$ (anti-C1q) and lupus nephritis can be observed. However, the predictive value of anti$\mathrm{C} 1 \mathrm{q}$ titres for a renal flare remains to be determined. Increasing titres of anti-C1q before the occurrence of clinical apparent nephritis might not only serve as a clinical parameter but also indicate a direct pathogenic mechanism of anti-C1q.
\end{abstract}

Methods. The aim of this study was to analyse the occurrence of anti-C1q before the onset of experimental lupus nephritis in MRL/MpJ +/+ mice and to correlate anti-C1q titres with the type and severity of glomerulonephritis (GN) developing at advanced age.

Results. As judged by a number of morphological and immunological analyses, GN in MRL/MpJ +/+ mice resembled human lupus nephritis and occurred in variable degrees of severity. We also observed an abundant and early presence of anti-C1q. However, anti-C1q neither correlated 
with overall survival nor with any histological marker of severity of GN.

Conclusions. The absence of a correlation between the presence of anti-C1q and the occurrence of experimental lupus nephritis contradicts the hypothesis that anti-C1q are pathogenic. However, different pathogenic mechanisms of experimental lupus nephritis and human proliferative lupus nephritis cannot be excluded.

Keywords: autoantibodies; complement; lupus nephritis

\section{Introduction}

In patients with systemic lupus erythematosus (SLE), a strong correlation between the occurrence of autoantibodies against $\mathrm{C} 1 \mathrm{q}$ (anti-C1q), the first component of the classical pathway of complement, and lupus nephritis has been demonstrated. Furthermore, a rise in antiC1q titre was suggested to be predictive for a renal flare. However, the predictive value of anti-C1q for severe lupus nephritis remains controversial $[1,2]$. Such an analysis in patients is limited by the difficulty of close follow-up in relation to biopsy-confirmed nephritis. In contrast, the analysis of lupus-prone mice for the presence of anti-C1q in relation to the occurrence of severe glomerulonephritis (GN) might allow the predictive value of anti-C1q to be determined.

Several mouse models of human SLE have been described. Although these models differ considerably from each other, they mostly share two phenotypic characteristics: the occurrence of autoantibodies and GN [3-5]. Among other autoantibodies, anti-C1q have been described in the three best-characterized lupus-prone strains: MRL/ MpJ-lpr/lpr, BXSB and (NZBxNZW) F1 [6-9]. The highest levels of anti-C1q have been detected in MRL/MpJ-lpr/lpr, and in this strain, anti-C1q were shown to be associated with low C1q levels [7]. However, this mouse model of SLE is not ideal to dissect the time course of the disease because of its fast and severe course. Furthermore, all MRL/MpJ-lpr/lpr mice had anti-C1q antibodies and all developed a severe and very early GN. Therefore, no clear correlation between anti-C1q and nephritis could be established [9].

In contrast, MRL/MpJ +/+ mice, which lack the lpr mutation spontaneously, develop an SLE-like autoimmune syndrome in a more delayed and less uniform fashion. In this strain, GN is a late event and occurs in variable degrees of severity. Although GN is considered to resemble human lupus nephritis, detailed histopathological analyses of this strain are scarce [10]. Lack of $\mathrm{C} 1 \mathrm{q}$ has been shown to accelerate the disease $[11,12]$, supporting the hypothesis that anti-C1q play a role in the pathogenesis of GN in MRL/ $\mathrm{MpJ}+/+$ mice. In addition, preliminary studies in a small cohort of MRL/MpJ +/+ mice had shown that some but not all of these mice developed elevated titres of anti-C1q early in life, when compared to normal BALB/c mice. Furthermore, anti-C1q could not be detected in MRL/MpJ +/+ mice being C1q-deficient (unpublished data). Thus, anti-
C1q associated with low C1q could be involved in the pathogenesis of murine $\mathrm{GN}$ in a disease-modifying way.

To test the predictive value of anti-C1q antibodies and the hypothesis that these have a pathogenic role in lupus nephritis, we followed MRL/MpJ +/+ mice and analysed potential correlations between anti-C1q titres before the onset of nephritis and the final severity of GN.

The aims of the presented study were (i) to give a detailed morphological description of the glomerular histopathology of autoimmune MRL/MpJ +/+ mice and (ii) to analyse the occurrence of anti-C1q before the onset of nephritis and to correlate anti-C1q titres with the type and severity of GN.

\section{Materials and methods}

\section{Animals and experimental protocol}

Female MRL/MpJ +/+ mice were obtained from the Jackson Laboratory (Bar Harbor, ME, USA) at 4 weeks of age. Control BALB/c mice were maintained at our animal facility. All animals had free access to water and standard chow. Animal care and experimentation were performed in accordance with the national guidelines (Federal Veterinary Office) for the care and use of laboratory animals. Serum and urine were first collected every month, then every second month. Mice were euthanized according to the national guidelines for the care and use of laboratory animals with a carbon dioxide chamber or with pentobarbital followed by axle bleeding and collection of kidneys for further analysis.

Three cohorts of $30 \mathrm{MRL} / \mathrm{MpJ}+/+$ mice each were analysed. In the first cohort, the survival of MRL/MpJ +/+ mice was determined and compared to the survival of $12 \mathrm{BALB} / \mathrm{c}$ control mice. In the second and third cohorts, MRL/MpJ +/+ mice were euthanized at 11 and 14 months in order to analyse the degree of GN at these ages. As a control, kidneys and spleens from 6-week-old and 14-month-old BALB/c mice were used.

\section{Morphological studies}

All morphological studies were performed with histological sections of 11- and 14-month-old MRL/MpJ +/+ mice. Eleven-month-old mice had only mild nephritis which didn't allow a distinct grading required for the correlation with anti-C1q titres. Therefore, detailed morphological analyses were only performed in 14-month-old mice.

Light microscopy. The kidneys were divided lengthwise. A slice of kidney was fixed in $4 \%$ phosphate-buffered formalin and then embedded in paraffin. Three-micrometre-thick sections were stained with H\&E, PAS, trichrome (chromotrope aniline blue) and methenamine silver.

Electron microscopy. Remaining tissue was used for electron microscopic studies. Fixation was done in 3\% phosphate-buffered glutaraldehyde, embedding in epon. Ultra-thin sections were stained with osmium tetroxide and contrasted with lead citrate.

Morphometry. Unstained paraffin sections were mounted with a medium containing Hoechst blue (H33258). Photographs of 10 consecutive glomeruli of each mouse were taken under fluorescent light using a DAPI filter as well as under visible light using phase contrast with a Zeiss microscope equipped with an Axiocam. The Hoechst blue-positive nuclei were counted manually in each glomerular cross section. The area of a glomerular cross section was determined in the phase contrast photographs using the Zeiss Axiovision AutoMeasure module.

Light microscopic evaluation. The following morphological findings were systematically assessed by light microscopy and graded 0-3+ (absentsevere): mesangial matrix expansion, mesangial hypercellularity, intracapillary hypercellularity, lobulation of the glomerular tuft, mesangial and peripheral protein deposits (as seen with trichrome stain) and active lesions (mesangiolysis, tuft necrosis, fibrin exudation into the capsular space, crescents, protein thrombi, vasculitis). The total score could reach a max- 


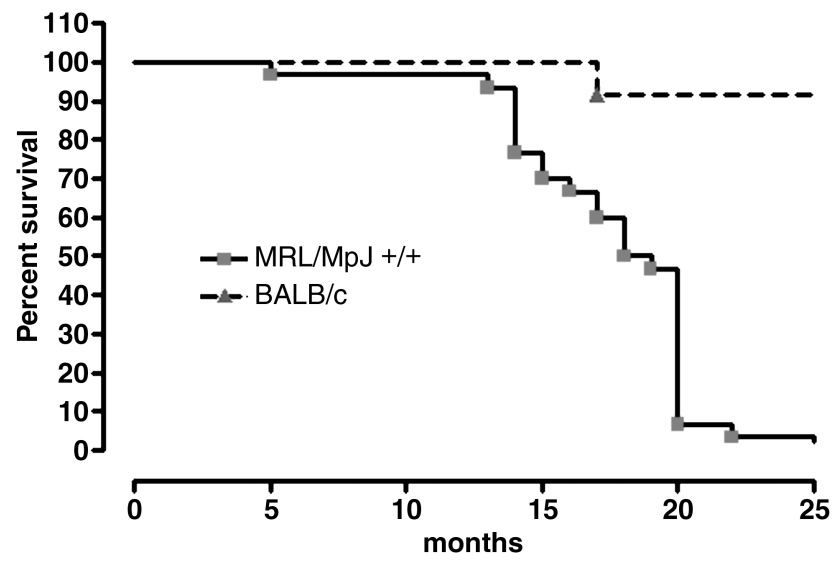

Fig. 1. Survival analysis of $M R L / M p J+/+$ and $B A L B / c$ mice. By 15 months the mortality rate in the MRL/MpJ +/+ group was $30 \%$ compared with no mortality observed in the BALB/c wild-type group. (By 22 months the mortality rate in the MRL/MpJ +/+ group was $100 \%$ compared to $8.33 \%$ in the $\mathrm{BALB} / \mathrm{c}$ wild-type controls). The survival curves differed significantly (by log-rank test, $\mathrm{P}<0.0001$ ).

imum of 18 (range $0-18$ ). In addition, the following features were recorded as present/not present: obsolescent glomeruli, arteriolar hyalinosis and lymph follicules.

For statistical evaluation, three groups each containing seven animals with increasing scores $(0-4,5-9,10-18)$ were used.

\section{Immunohistochemistry}

To reveal IgG and C3 deposition, sections were deparaffinated and hydrated according to standard protocols. Sections were digested with protease XXIV $(0.03 \% 50 \mu \mathrm{L}$ per section) (Sigma, Missouri, USA) for $5 \mathrm{~min}$ (IgG) and $10 \mathrm{~min}(\mathrm{C} 3)$ and washed in $100 \%$ ethanol. Then they were rehy- drated in PBS and blocked with normal goat serum (Vector Laboratories, Burlingame, CA). Sections were incubated overnight in PBS, 1\% BSA with biotinylated goat anti-ms IgG 1/1000 (SouthernBiotech, Alabama USA) and rabbit anti-human C3c 1/2000 (DakoCytomation, Glostrup, Denmark), which crossreacts with mouse C3c. After washing in PBS, sections were incubated for $45 \mathrm{~min}$ in PBS 1\% BSA with biotinylated goat anti-rabbit IgG (Vector Laboratories, Burlingame, CA). They were washed again and incubated with VECTASTAIN Elite ABC reagent (Vector Laboratories, Burlingame, CA) for $30 \mathrm{~min}$, then washed again and incubated with freshly prepared DAB solution (Vector Laboratories, Burlingame, CA) for 2 min until a suitable colour had developed. Counterstaining was carried out with Mayer's haematoxylin (J.T. Baker, Mallinckrodt Baker, Inc., Philipsburg, NJ, USA) for 2 min followed by 2 min each of rinsing in tap water, distilled water, ethanol 70\%, 96\%, 100\% and UltraClear (J.T. Baker, Mallinckrodt Baker, Inc., Philipsburg, NJ, USA). Finally, samples were mounted with UltraKitt mounting medium (J.T. Baker, Mallinckrodt Baker, Inc., Philipsburg, NJ, USA). For staining of nuclei, sections were deparaffinated and hydrated, followed by staining with bisbenzimide $\mathrm{H} 33258$ fluorochrome trihydrochloride (Calbiochem, Merck, Darmstadt, Germany) for $1 \mathrm{~min}$. Then sections were washed three times and mounted with Ultrakitt mounting medium (J.T. Baker, Mallinckrodt Baker, Inc., Philipsburg, NJ, USA). The degree of IgG and C3 deposition was systematically assessed by light microscopy and graded $0-3+$.

Glomerular T cells were stained using deparaffinated and hydrated paraffin sections that were blocked with normal goat serum and incubated with a polyclonal rabbit anti-mouse CD3 antibody (Dako Denmark A/S, Glostrup, Denmark). After washing, the binding of the primary antibody was revealed by a biotinylated goat anti-rabbit IgG antibody (Vector Laboratories, Burlingame, CA) followed by incubation with VECTASTAIN Elite ABC reagent (Vector Laboratories, Burlingame, CA) for $30 \mathrm{~min}$, AEC Substrat-Chromogen (Dako Denmark A/S, Glostrup, Denmark) and Mayer's haematoxylin counterstaining (J.T. Baker, Mallinckrodt Baker, Inc., Philipsburg, NJ, USA) as outlined before. In analogy, glomerular macrophage staining was performed using a rat anti-mouse Mac2 antibody (Cedarlane Laboratories, Hornby, Ontario, Canada) revealed by a biotinylated secondary goat anti-rat IgG antibody (Southern Biotechnology, Birmingham, AL, USA). For both, numbers of cells per glomerulus were expressed as the median count of 50 glomeruli.

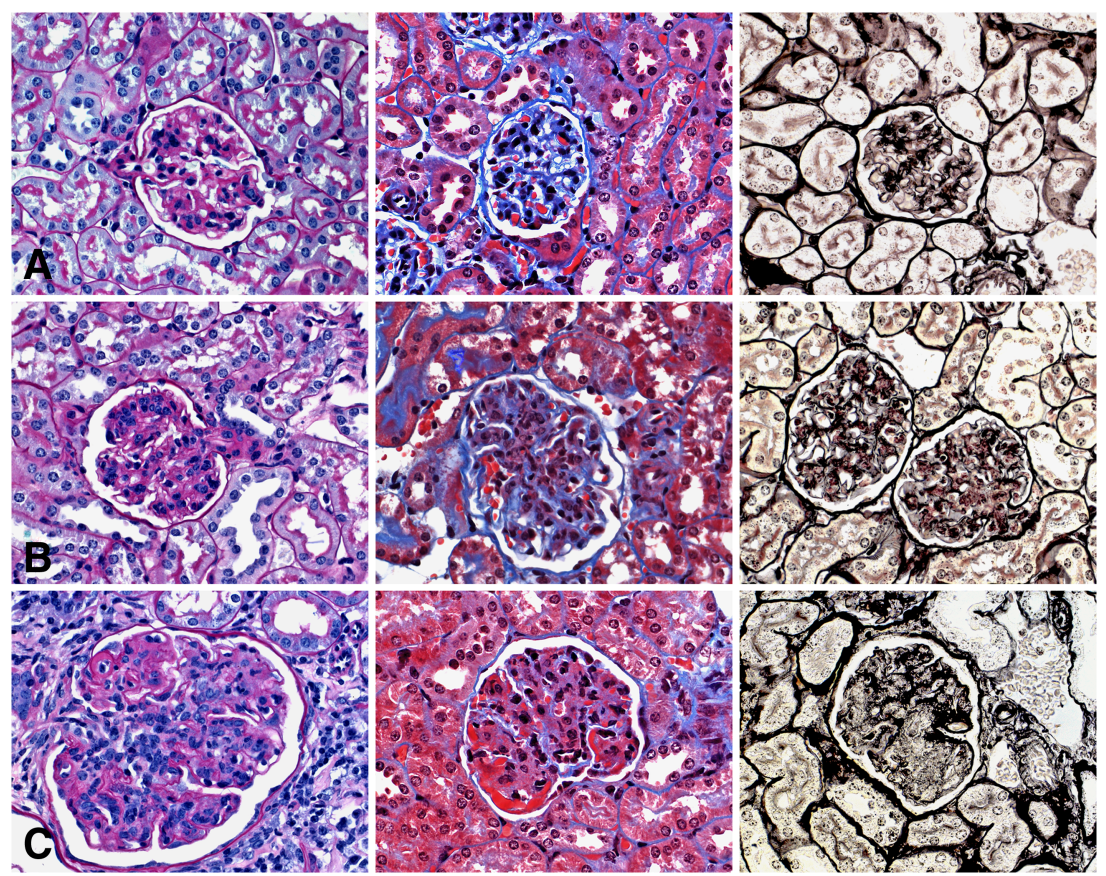

Fig. 2. Typical morphological lesions in MRL/MpJ +/+ mice of Groups I (A), II (B) and III (C) by light microscopy (LM $\times 600)$, in the PAS, trichrome and silver stain (from left to right). a. Mild mesangial expansion and hypercellularity in the mesangium. b. Moderate mesangial expansion and hypercellularity in the mesangium and few capillary loops. c. Severe lobulation of the glomeruli with prominent mesangial expansion and mesangial as well as intracapillary hypercellularity. Protein deposits and thrombi (reddish/red) in the PAS and trichrome stain. Severe mesangiolysis in the silver stain. Note increase of glomerular size from (A) to (C). 


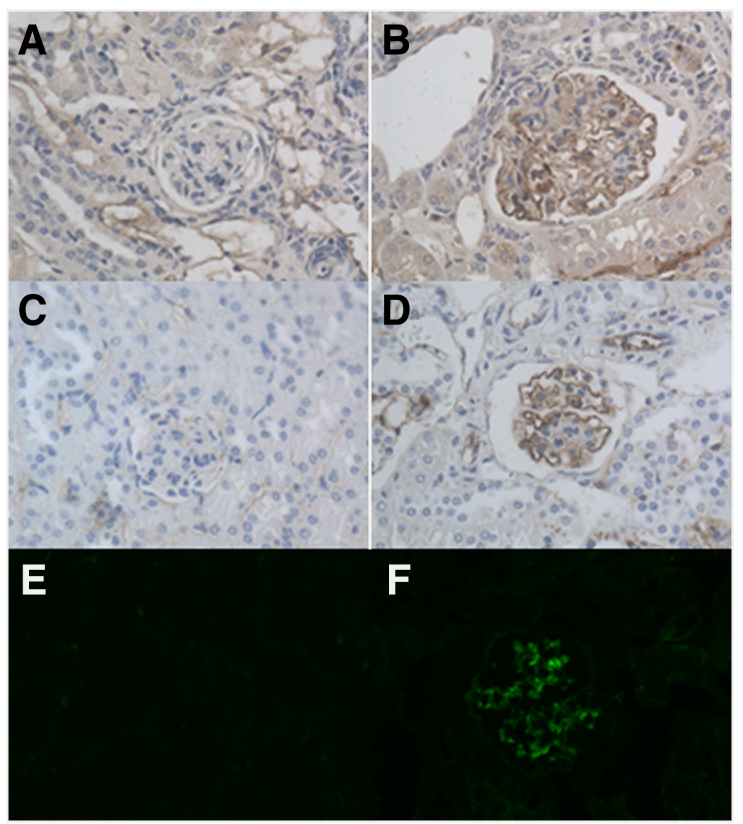

Fig. 3. Deposition of $\mathrm{C} 3, \mathrm{IgG}$ and $\mathrm{C} 1 \mathrm{q}$ in renal sections of $\mathrm{MRL} / \mathrm{MpJ}+/+$ mice. MRL/MpJ +/+ mice having deposition of $\mathrm{C} 3, \mathrm{IgG}$ and/or $\mathrm{C} 1 \mathrm{q}$ varied in their positivity, whereas others were negative. (A) Glomerulus of a MRL/MpJ +/+ mouse, no deposition of C3. (B) Deposition of C3 in a glomerulus of another mouse. (C) Glomerulus without IgG deposition. (D) Glomerular deposition of IgG. (E) Glomerulus of a MRL/MpJ +/+ mouse, no deposition of C1q. (F) Deposition of C1q in a glomerulus of another mouse. Original magnification $\times 40$.

\section{Immunofluorescence}

Cryostat sections of $4 \mu \mathrm{m}$ of snap-frozen kidneys were stained for the deposition of C1q. Sections were blocked with $5 \%$ normal rat serum (Sigma-Aldrich, St. Louis, MO, USA) in PBS for $30 \mathrm{~min}$, then incubated with FITC-labelled rat anti-mouse C1q (Cedarlane, Burlington, Canada) diluted $1 / 10$ in PBS for $60 \mathrm{~min}$. Sections were washed three times using PBS then mounted with UltraKitt mounting medium (J.T. Baker, Mallinckrodt Baker, Inc., Philipsburg, NJ, USA). Grading of immunofluorescence was as follows: 1 = focal, 2 = diffuse weak and $3=$ diffuse strong. For evaluation purposes, two groups were formed according to the degree of complement deposition (score: $<=2 />2$ ).

Stainings for complement IgG and $\mathrm{C} 3$ were performed in analogy using normal goat serum for blocking followed by a FITC-labelled poly- clonal goat anti-mouse IgG (Sigma, St. Louis, Missouri, USA) and goat anti-mouse C3 (ICL, Newberg, OR, USA), respectively.

\section{Detection of total $\operatorname{IgG}$, autoantibodies against complement $\mathrm{Cl} q$ and anti-nuclear antibodies}

For the detection of murine anti-C1q, a highly significant correlation of results was obtained comparing assays in which purified human $\mathrm{C} 1 \mathrm{q}$ or purified mouse C1q was used as the antigen [6]. Therefore, ELISA plates (Nunc, Rosklide, Denmark) for anti-C1q measurements were coated overnight at $4^{\circ} \mathrm{C}$ with purified human $\mathrm{Clq}$ (gift from Bühlmann Laboratories, Schönenbuch, Switzerland; $>99 \%$ pure as judged by SDS-PAGE) at a concentration of $0.5 \mu \mathrm{g} / \mathrm{well}$ or for determination of total IgG with goat anti-ms Ig $(\mathrm{H}+\mathrm{L})$ (SouthernBiotech, Alabama, USA) at a concentration of $2 \mu \mathrm{g} / \mathrm{mL}$. For the anti-C1q ELISA, serum samples were diluted 1:50 in PBS Tween $(0.05 \%), 1 \% \mathrm{FCS}$ containing $1 \mathrm{M} \mathrm{NaCl}$. For the detection of $\mathrm{IgG}$, serum samples were diluted $1 / 800000$ in PBS. After incubation with serum samples, plates were washed and bound $\operatorname{IgG}$ was detected using biotinylated polyclonal goat anti-mouse IgG (SouthernBiotech, Alabama, USA) and horseradish peroxidase-labelled Streptavidin (Jackson ImmunoResearch Europe, Suffolk, UK). A monoclonal mouse anti-human $\mathrm{C} 1 \mathrm{q}$ (generated by immunization of $\mathrm{Clq}_{\mathrm{a}}$-deficient mice, clone 23D11) [13] was used to generate a standard curve. Anti-C1q are expressed as units per millilitre. To calculate the amount of $\mathrm{IgG}$, a standard mouse IgG preparation (SouthernBiotech, Alabama, USA) was used.

Anti-nuclear antibodies (ANA) were determined as described previously [14] (with the kind help of Prof. Rolink, Basel). In short, snap-frozen sections of kidneys from RAG-2-/- mice were incubated with sera diluted 1:20 and bound antibodies revealed with an FITC-labelled anti-mouse IgG antibody.

\section{Proteinuria, haematuria and serum creatinine}

Urine was regularly collected and analysed using dipsticks (Multistix ${ }^{\circledR} 5$ SG, Bayer Diagnostics, Bridgend, UK) and a urine chemistry analyser (Clinitek 50, Bayer Diagnostics, Bridgend, UK). Serum creatinine was measured by quantitative colorimetric determination at $510 \mathrm{~nm}$ using a commercially available assay (Creatinine Assay Kit, Biochain, Hayward, CA, USA).

\section{Antibody elution from kidneys}

The procedure was performed as described before [9]. In short, six kidneys from six mice with high titres of anti-C1q antibodies were pooled, minced and collected in $3 \mathrm{~mL}$ PBS containing protease inhibitor cocktail (Roche, Mannheim, Germany). The mixture was centrifuged at $3000 \mathrm{rpm}$ for $5 \mathrm{~min}$ and supernatants were collected. Pellets were washed and resuspended in $1.5 \mathrm{~mL}$ elution buffer consisting of $0.1 \mathrm{M}$ glycine- $\mathrm{HCl}, 0.15 \mathrm{M} \mathrm{NaCl}, \mathrm{pH}$ 2.5 and sonicated on ice with three bursts of $30 \mathrm{~s}$ and amplitude of 25 . After overnight rotation at $4{ }^{\circ} \mathrm{C}$, samples were centrifuged for $10 \mathrm{~min}$ at 10 $000 \mathrm{rpm}$ and supernatants were collected and adjusted to $\mathrm{pH}$ 7.5. Samples

Table 1. Light microscopical findings and immune deposits in three groups of animals with different degrees of morphological lesions

\begin{tabular}{|c|c|c|c|c|}
\hline Parameter, median (range) & Group I & Group II & Group III & P-value \\
\hline Total score & $3(2-4)$ & $6(5-8)$ & $12(9-15)$ & 0.0001 \\
\hline Mesangial enlargement & $1(1-2)$ & $2(2-2)$ & $2(2-3)$ & 0.0007 \\
\hline Mesangial proliferation & $1(1-2)$ & $2(2-2)$ & $3(2-3)$ & 0.0033 \\
\hline Intracapillary hypercellularity & $0(0-1)$ & $1(0-1)$ & $2(1-3)$ & 0.0010 \\
\hline Lobulation & $0(0)$ & $0(0-2)$ & $2(2-3)$ & 0.0004 \\
\hline Protein deposits & $0(0-1)$ & $0(0-2)$ & $1(1-3)$ & 0.0114 \\
\hline Glomerular area $\left(\times 10^{3} \mu \mathrm{m}^{2}\right)$ & $24.6(16.2-29.5)$ & $29.1(23.1-32.4)$ & $33.7(25.6-39.4)$ & 0.0043 \\
\hline Glomerular DAPI+ area $\left(\times 10^{3} \mu \mathrm{m}^{2}\right)$ & $9.1(5.6-13.4)$ & $11.0(9.0-12.4)$ & $12.7(11.1-13.7)$ & 0.0091 \\
\hline Glomerular DAPI+ area per glomerular area & $0.37(0.34-0.42)$ & $0.37(0.33-0.43)$ & $0.39(0.30-0.46)$ & 0.7799 \\
\hline Glomerular CD3+ cells & $5.4(3.1-6.6)$ & $6.0(4.3-9.2)$ & $8.1(6.4-9.8)$ & 0.0113 \\
\hline Glomerular Mac $2+$ cells & $0.7(0.2-2.4)$ & $3.5(0.6-6.5)$ & $5.3(4.8-6.8)$ & 0.0008 \\
\hline Glomerular IgG deposits & $0(0-2)$ & $0(0-2)$ & $0(0-2)$ & 0.9823 \\
\hline Glomerular $\mathrm{C} 3$ deposits & $0(0-1)$ & $1(0-3)$ & $2(1-3)$ & 0.0079 \\
\hline Glomerular C1q deposits & $0(0-3)$ & $1(0-3)$ & $1(0-3)$ & 0.1726 \\
\hline
\end{tabular}




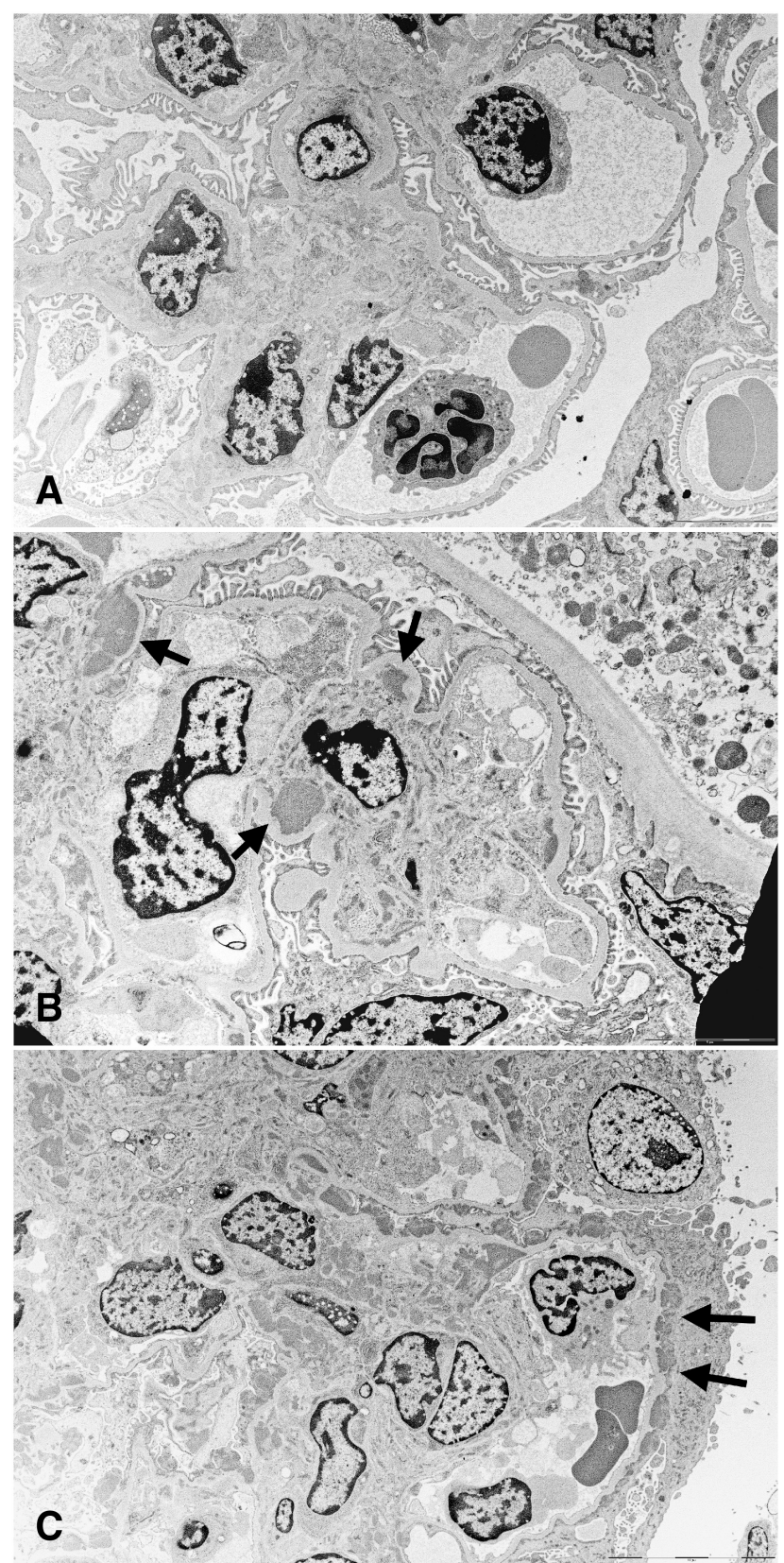

Fig. 4. Typical morphological lesions in MRL/MpJ $+/+$ mice of Groups I (A), II $(\mathbf{B})$ and III $(\mathbf{C})$ by electron microscopy $(\mathrm{EM} \times 3500)$. a. Slight mesangial expansion due to matrix increase and minute mesangial electron-dense deposits (not seen at this magnification), no cell increase. Basement membranes unchanged. One polymorphonuclear leukocyte in the capillary lumen. No foot process fusion of podocytes. b. Very similar morphology as compared with (A), but large and lumpy electron-dense deposits in the mesangium (arrow). c. In addition to (B), numerous electron dense deposits of variable size on the outside of the basement membrane (arrow). Complete foot process effacement of podocytes.

were then tested by ELISA for the presence of anti-C1q and IgG as described above.

\section{Statistics}

Kaplan-Meier curves for the analysis of survival curves, column statistics, area under the anti-C1q curves (AUC anti-C1q), non-parametric correlation tests (Spearman) and Mann-Whitney $U$-test or Kruskal-Wallis tests for the comparison of multiple groups were performed where appro-
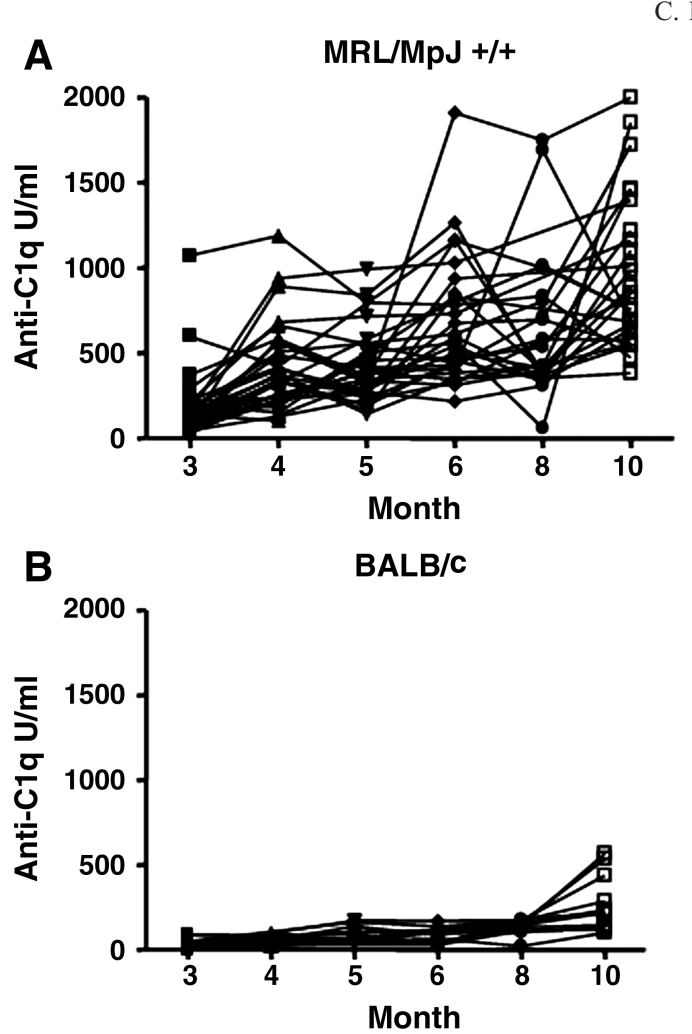

Fig. 5. Anti-C1q autoantibody titres in individual mice over time. (A) $\mathrm{MRL} / \mathrm{MpJ}+/+$ and (B) BALB/c mice. Data are expressed relative to a standard monoclonal mouse anti-C1q antibody. In MRL/MpJ +/+ mice, anti-C1q levels strongly varied over time.

priate. Levels of severity of complement deposition were analysed by Chisquare tests and mortality by log-rank test. All statistics were performed using GraphPad Prism version 4 (GraphPad Software, San Diego, USA).

\section{Results}

\section{Survival analysis}

First, a survival study of $30 \mathrm{MRL} / \mathrm{MpJ}+/+$ and $12 \mathrm{BALB} / \mathrm{c}$ control mice was performed. The onset of death within the $\mathrm{MRL} / \mathrm{MpJ}+/+$ group occurred after 5 months, and 50\% mortality was reached at 18 months. After 22 months all $\mathrm{MRL} / \mathrm{MpJ}+/+$ mice were dead. At this time, mortality in the BALB/c control mice group was $8.33 \%$ (1 out of 12 ). The difference in mortality between the two mouse strains was significant (by log-rank test, $\mathrm{P}<0.0001$ ) (Figure 1).

\section{Renal pathology}

Since 11-month-old mice had only mild nephritis, all the following morphological analyses were only performed in 14-month-old mice. At this age, $95 \%$ of MRL/MpJ +/+ mice were ANA positive.

Light microscopy. At low magnification, the basic structure of the kidneys was well preserved. In the cortex, enlarged glomeruli were apparent exhibiting variable degrees of hypercellularity. Occasional $(<5 \%)$ obsolescent glomeruli were seen in practically all mice. The tubulointerstitial space was unremarkable, apart from variable 


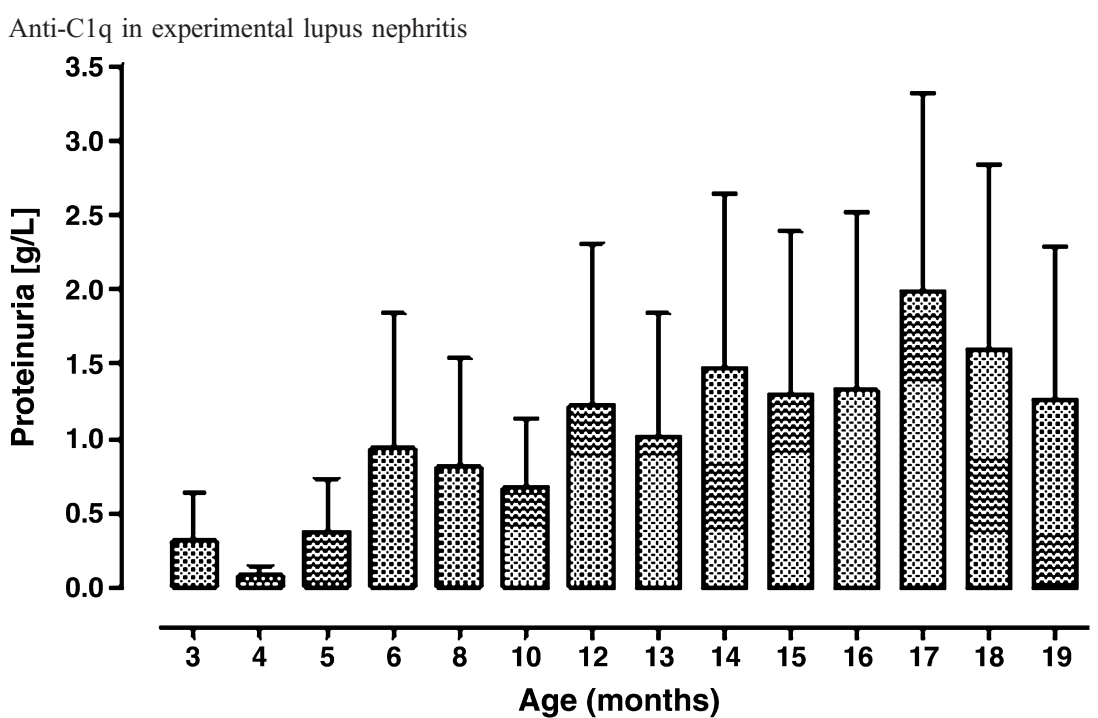

Fig. 6. Proteinuria in ageing $\mathrm{MRL} / \mathrm{MpJ}+/+$ mice. Proteinuria in $\mathrm{MRL} / \mathrm{MpJ}+/+$ mice was determined using dipstick analyses. Data are shown as mean $+/-$ standard deviation.

numbers of cortical and medullary lymph follicles seen in most animals. Individual mice exhibited small foci of interstitial plasma cell aggregates.

Hyalinosis was rare in the arterioles. The arteries (with two exceptions) and the veins were unremarkable. At higher magnification, a variable degree of mesangial expansion was seen, in part due to an increase of the mesangial matrix and partly due to a highly variable hypercellularity or both. Protein deposits were visible by trichrome stain in the mesangium and less frequently in the periphery. This basic picture of glomerular injury had several variants. The more severe the mesangial hypercellularity, the more frequent was a generally segmental intracapillary hypercellularity due to mononuclear cells. In cases of particularly severe hypercellularity, the glomeruli were often segmentally or globally lobulated. The BALB/c control mice studied in the same way showed no pathology at all.

In addition, occasional so-called active lesions were found, in the form of segmental tuft necrosis, segmental proliferative or proliferative sclerosed crescents, mesangiolysis, protein thrombi or vasculitis. These active lesions were extremely rare and involved at most $1 \%$ of the glomeruli. The peripheral loops were occasionally thickened, but clear cut doubling of the basement membrane with mesangial interposition was not observed (Figure 2).

Immunohistology. In immunohistochemical stainings, mesangial, and more rarely peripheral, deposits of varying intensity were seen for complement C3 (62\%) and $\mathrm{IgG}$ (29\%). Using immunofluorescence, C3 and IgG stained positive in 94 and $100 \%$ of mice, respectively. C1q positivity was found in $71 \%$ of mice where weakly positive findings dominated (Figure 3).

Semi-quantitative and quantitative evaluation. The arbitrary assignment of the animals into three groups according to increasing scores of injury reflects the level of variability and the pattern of injury (Table 1).
Group 1: The group with the lowest injury score is essentially distinguished by a slight increase in mesangial matrix and cell number, without regular presence of immunoglobulin or complement deposits. The basic pattern corresponded to minor glomerular abnormalities or mild mesangial proliferative GN.

Group II: A marked increase in mesangial matrix and cell number is accompanied by mild segmental intracapillary hypercellularity. Cell proliferation paralleled the increase in size (glomerular DAPI+ area/glomerular area). Slight C3 or C1q deposits could be seen. The lesions seen corresponded to severe mesangio-proliferative GN.

Group III: In this group with the highest injury scores, the mesangial changes and the segmental, intracapillary hypercellularity were even more prominent than in group II. Protein deposits were seen with the trichrome stain, identified as complement deposits (C3 and/or C1q) by immunofluorescence. Quantitative evaluation showed, in addition to a further increase in glomerular cell size and number, an over proportional cell proliferation (glomerular DAPI+ area/glomerular area). The intracapillary hypercellularity was mainly due to an increase in monocytes more than lymphocytes (see Table 1). All cases with so-called active lesions (see above) were found in group III. The pattern of injury corresponded to a diffuse proliferative GN with active lesions.

The level of severity of the GN correlated with the level of complement deposition (C3) seen by light microscopy $(\mathrm{P}=0.0079)$.

Electron microscopy. Group I and II: Both groups of mice exhibited mesangial enlargement resulting from a modest increase in matrix material and prominent mesangial cell activation. The mice of group I revealed numerous small, osmiophilic deposits along the para-mesangial basement membrane, in the sample from group II deposits were large and lumpy. There were no deposits in the periphery and no fusion of the podocyte foot processes. 
A

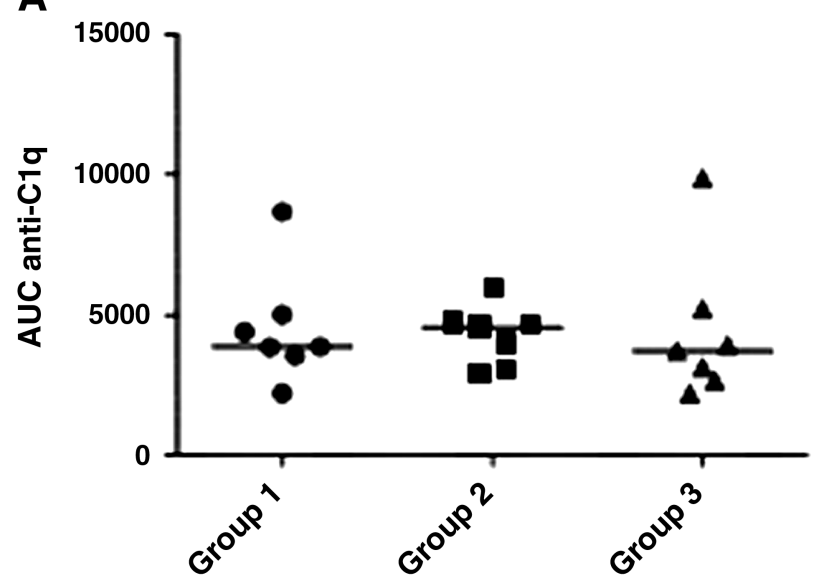

B

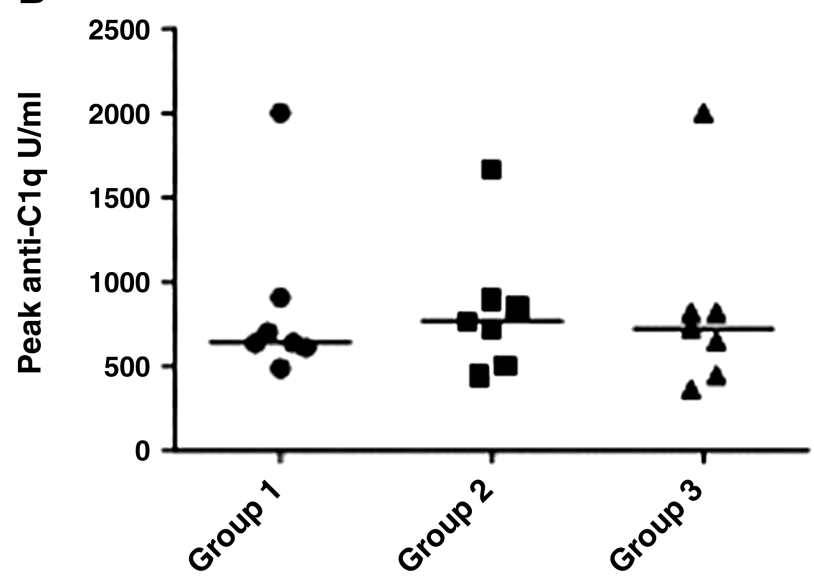

C

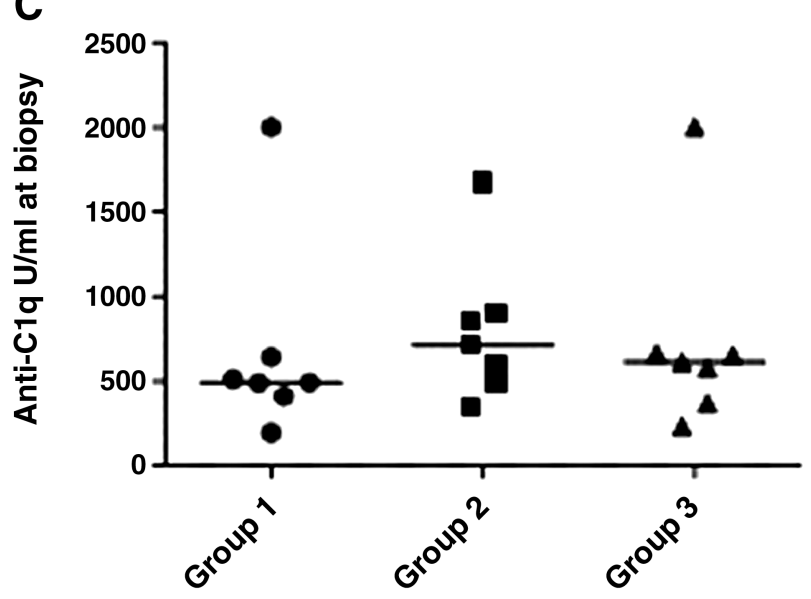

Fig. 7. Correlation between anti-C1q autoantibodies and renal damage. Mice were divided into three groups (Groups 1-3) according to renal pathology and analysed for their correlation with anti-C1q autoantibodies. No significant difference could be found between the three groups when looking at areas under the anti-C1q curves (AUC anti-C1q), peak levels of anti-C1q or titres at time of biopsy. Medians are shown as horizontal lines.

Group III: These mice had massive mesangial expansion, due to cell proliferation and cell activation, modest matrix expansion and massive accumulation of osmiophilic deposits of varying size. In addition, subepithelial deposits of varying size irregularly distributed along the peripheral basement membrane were seen, in part flanked by spikes. The podocytes were highly activated and exhibited complete fusion of the foot processes. Subendothelial deposits were comparatively small and rarer. In the endothelium, tubulo-reticular or fingerprint-like structures could not be found in any animal. The vessels and the tubulo-interstitial space were unremarkable, in particular no osmiophilic deposits were detected along the peripheral capillaries (Figure 4).

\section{Detection of anti-Clq autoantibodies in serum}

Already at 3 months of age, most MRL/MpJ +/+ mice had elevated anti-C1q when compared to BALB/c control mice. Although all mice showed a rise in titre at later time-points, individual mice showed a high variability in anti-C1q titres (Figure 5). No correlation between survival and either peak anti-C1q levels or areas under the anti-C1q curves (AUC anti-C1q) was found.

\section{Correlation between anti-Clq autoantibodies and renal damage}

Using dipstick analyses, we observed a progressive increase in proteinuria (Figure 6) but did not detect a significant haematuria in ageing MRL/MpJ +/+ mice.

No correlation was found between degrees of proteinuria and serum creatinine concentrations (median $0.56 \mathrm{mg} / \mathrm{dL}$, range $0.39-1.41 \mathrm{mg} / \mathrm{dL}$ in 14-month-old MRL/MpJ +/+ mice) on the one hand and any parameter of anti-C1q, i.e. areas under the anti-C1q curves, the peak anti-C1q levels or the anti-C1q levels at the time of euthanasia, on the other hand.

According to the renal pathology, mice were separated in three groups (see above) and analysed for their correlation with anti-C1q autoantibodies. No significant differences could be found between the three groups with regard to the areas under the anti-C1q curves (AUC anti-C1q), the peak anti-C1q levels or the anti-C1q levels at the time of euthanasia (Figure 7). Furthermore, no correlations were found between AUC anti-C1q, peak anti-C1q or anti-C1q titres at the time of euthanasia and any parameter of renal pathology (studied by light microscopy or immunohistochemistry, Table 2).

\section{Detection of anti-C1q autoantibodies in kidney eluate}

The lack of correlation between anti-C1q autoantibodies and renal damage was not due to the lack of anti-C1q deposition in kidneys. Anti-C1q autoantibodies were detected in the eluate of kidney tissue from 14-month-old $\mathrm{MRL} / \mathrm{MpJ}+/+$ mice having high titres of anti-C1q, but not in eluates of kidney tissue from BALB/c control mice. Comparing anti-C1q levels in the eluate relative to eluted total IgG with corresponding serum anti-C1q levels relative to total serum IgG, a strong enrichment of anti-Clq in the kidney eluate could be seen of MRL/MpJ +/+ but not in $\mathrm{BALB} / \mathrm{c}$ mice (Figure 8). 
Table 2. No correlation of anti-C1q antibodies with parameters of glomerular histology in MRL/MpJ $+/+$ mice

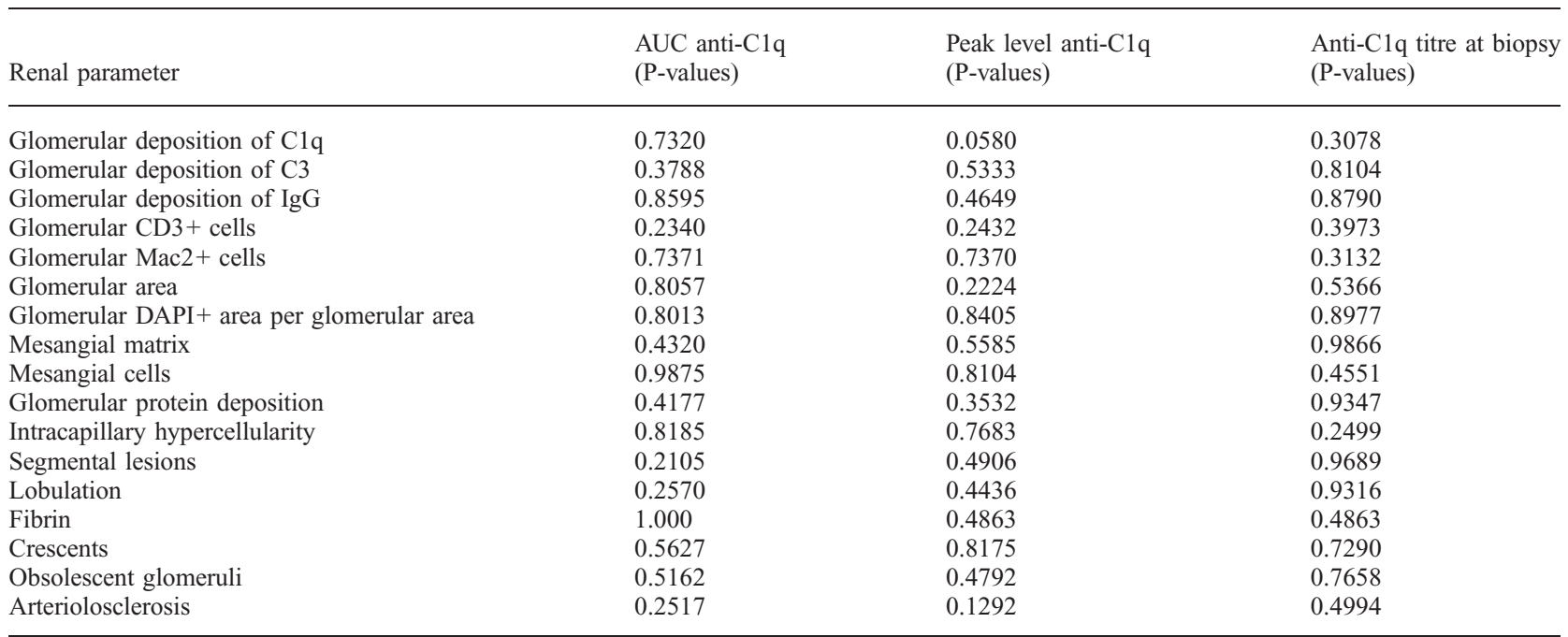

$\mathrm{AUC}=$ area under the anti-C1q curve.

\section{Discussion}

Our study provides a detailed morphological analysis of the lupus nephritis in MRL/MpJ +/+ mice but fails to demonstrate a correlation between anti-C1q antibodies and the severity of GN.

$\mathrm{MRL} / \mathrm{MpJ}+/+$ mice develop a highly variable picture of GN at 14 months which reflects the picture seen in human lupus nephritis. There is a range from minor glomerular lesions, with only tiny osmiophilic deposits in the mesangium without regular complement deposition, to mesangio-

MRL/MpJ +/+ 14 months anti-C1q/lgG BALB/c 14 months anti-C1q/lgG

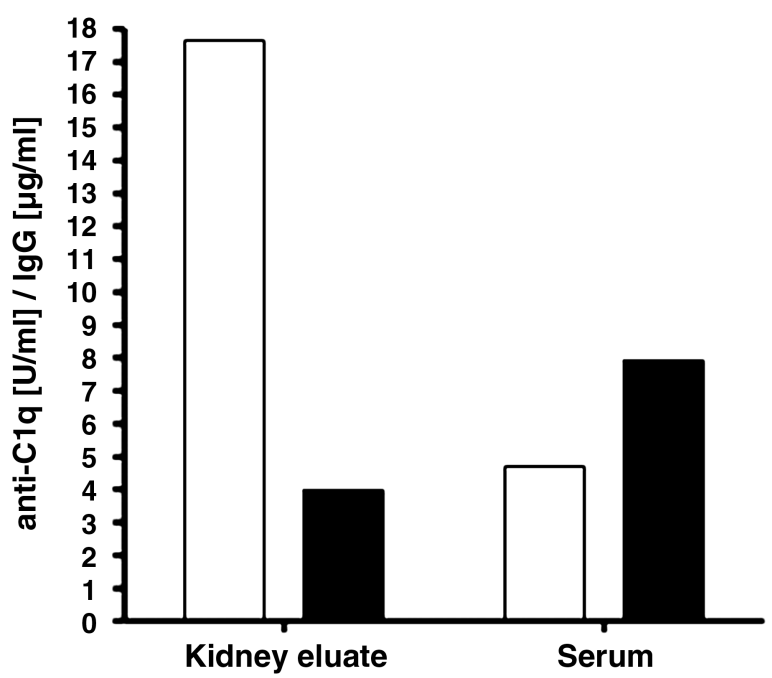

Fig. 8. Renal elution of anti-C1q antibodies. Anti-C1q reactivity per microgram IgG in eluate of kidney tissue and serum of 14-month-old $\mathrm{MRL} / \mathrm{MpJ}+/+$ mice compared to age-matched BALB/c control mice. An enrichment of anti-C1q autoantibodies is seen in the kidney eluate of $\mathrm{MRL} / \mathrm{MpJ}+/+$ but not in $\mathrm{BALB} / \mathrm{c}$ mice when compared to serum. proliferative GN with marked mesangial expansion and lumpy mesangial deposits and to diffuse proliferative GN which is typical for severe lupus nephritis in man. These latter cases have, in addition to marked mesangial expansion with cell proliferation, large, lumpy mesangial deposits accompanied by minor subendothelial deposits as well as in part massive subepithelial deposits and dominant complement deposition. However, the so-called active lesions were only observed in very few glomeruli. In most other mouse models of lupus nephritis (NZBxW, MRL/1, BXSB, MRL/MpJ-lpr/lpr), a lower degree in the variability of the GN severity is seen between individual animals. At any particular time point, and often early (3-6 months), a severe, homogenous GN of diffuse proliferative type can be observed [3,5,15, personal observations]. Occasionally, these lesions are accompanied by severe exudative changes with crescent formation and, after an observation period exceeding 1 year, extensive glomerular obsolescence. The latter type of changes were virtually absent in our study. In contrast to the other SLE mouse models, we did not observe regular IgG deposition in the MRL/MpJ +/+ model.

As described for other lupus-prone mouse strains, the $\mathrm{MRL} / \mathrm{MpJ}+/+$ mice developed elevated levels of antiC1q antibodies early in life. Levels of anti-C1q antibodies varied which allowed the analysis of differences between mice with high levels of anti-C1q and mice with low levels of anti-C1q. Fourteen-month-old mice with high titres of anti-C1q antibodies in serum had an enrichment of anti$\mathrm{Clq}$ in their kidneys. This finding is in line with the finding of anti-C1q antibodies in renal tissue of MRL/MpJ-lpr/lpr mice [9] as well as in post-mortem material of end-stage kidneys from patients with lupus nephritis [16]. At the age of 14 months some MRL/MpJ +/+ mice exhibited GN with increased glomerular cellularity as well as glomerular IgG, C1q and/or C3 deposition. This is in accordance with previous studies showing glomerular deposition of C3 and IgG starting already at 7 months $[11,17]$.

However, we found no correlation between levels of anti$\mathrm{C} 1 \mathrm{q}$ and any parameter of glomerular inflammation. In 
addition, levels of anti-C1q did not correlate with the overall survival of MRL/MpJ +/+ mice. Although we cannot exclude a role for deposited antibodies, our observational data suggest that anti-C1q are not involved in the pathogenic mechanism of GN in lupus-prone MRL/MpJ +/+ mice and do not support the hypothesis that anti-C1q have a pathogenic role in SLE. The finding is also in conflict with data from Trouw et al. showing that the injection of a monoclonal anti-C1q in mice pretreated with subnephritogenic doses of C1q-fixing anti-glomerular basement membrane antibodies resulted in exacerbation of the subclinical renal disease [18]. In another study, the injection of polyclonal rabbit anti-mouse $\mathrm{C} 1 \mathrm{q}$ into healthy mice resulted in glomerular complement activation, leukocyte influx and mild albuminuria [19]. As a consequence, we think that the type of GN might be critical in the determination of the predictive value and pathogenic role of anti-C1q. Independently, it has been recognized that translation of results obtained in experimental autoimmune diseases into the human situation is difficult [20]. Thus, in spite of morphological similarities, different mechanisms might be involved between the pathogenesis of human lupus nephritis and the GN seen in MRL/MpJ +/+ mice. Only large studies on SLE patients that are closely followed over long periods might provide a definitive answer to the question of the true predictive value of anti-C1q for renal flares.

In conclusion, we did not observe a correlation of anti$\mathrm{C} 1 \mathrm{q}$ with the survival or the severity of GN in lupus-prone mice. Therefore, our data do not support the hypothesis that anti-C1q have a pathogenic role in SLE. However, different pathogenic mechanisms might be involved in the GN of lupus-prone MRL/MpJ +/+ mice and human proliferative lupus nephritis.

Acknowledgements. We thank Mrs Brigitte Schneider, Mrs Ursula Duermueller and Mrs. Hedwig Niederer for their important help in the handling of animals and in the histopathological analyses. The study was supported by a SCORE fellowship from the Swiss National Foundation (3232BO-107248/2)

Conflict of interest statement. None declared.

\section{References}

1. Moroni G, Trendelenburg M, Del Papa N et al. Anti-C1q antibodies may help in diagnosing a renal flare in lupus nephritis. Am J Kidney Dis 2001; 37: 490-498

2. Grootscholten C, Dieker JWC, McGrath FD et al. A prospective study of anti-chromatin and anti-C1q autoantibodies in patients with proliferative lupus nephritis treated with cyclophosphamide pulses or azathioprine/methylprednisolone. Ann Rheum Dis 2007; 66: 693-696

3. Theofilopoulos AN, Dixon FJ. Murine models of systemic lupus erythematosus. Adv Immunol 1985; 37: 269-390

4. Peutz-Kootstra CJ, de Heer E, Hoedemaeker PJ et al. Lupus nephritis: lessons from experimental animal models. J Lab Clin Med 2001; 137 : 244-260

5. Andrews BS, Eisenberg RA, Theofilopoulos AN et al. Spontaneous murine lupus-like syndromes. Clinical and immunopathological manifestations in several strains. J Exp Med 1978; 148: 1198-1215

6. Hogarth MB, Norsworthy PJ, Allen PJ et al. Autoantibodies to the collagenous region of $\mathrm{C} 1 \mathrm{q}$ occur in three strains of lupus-prone mice. Clin Exp Immunol 1996; 104: 241-246

7. Trinder PK, Maeurer MJ, Schorlemmer HU et al. Autoreactivity to mouse C1q in a murine model of SLE. Rheumatol Int 1995; 15: $117-120$

8. Uwatoko S, Mannik M, Oppliger IR et al. C1q-binding immunoglobulin $\mathrm{G}$ in MRL/1 mice consists of immune complexes containing antibodies to DNA. Clin Immunol Immunopathol 1995; 75: 140-146

9. Trouw LA, Seelen MA, Visseren R et al. Anti-C1q autoantibodies in murine lupus nephritis. Clin Exp Immunol 2004; 135: 41-48

10. Hewicker M, Kromschröder E, Trautwein G. The pathogenesis of glomerulonephritis in MRL mice - murine lupus erythematosus. Verh Dtsch Ges Pathol 1989; 73: 129-132

11. Mitchell DA, Pickering MC, Warren J et al. C1q deficiency and autoimmunity: the effects of genetic background on disease expression. J Immunol 2002; 168: 2538-2543

12. Trendelenburg M, Manderson AP, Fossati-Jimack L et al. Monocytosis and accelerated activation of lymphocytes in C1q-deficient autoimmune-prone mice. Immunology 2004; 113: 80-88

13. Bigler C, Schaller M, Perahud I et al. Autoantibodies against complement $\mathrm{C} 1 \mathrm{q}$ specifically target $\mathrm{C} 1 \mathrm{q}$ bound on early apoptotic cells. J Immunol 2009; 183: 3512-3521

14. Bénard A, Ceredig R, Rolink AG. Regulatory T cells control autoimmunity following syngeneic bone marrow transplantation. Eur J Immunol 2006; 36: 2324-2335

15. Gunn HC, Ryffel B. Glomerulonephritis in NZB/W mice: therapeutic effect of cyclosporine. Clin Nephrol 1986; 25: 1189-192

16. Mannik M, Wener M. Deposition of antibodies to the collagen-like region of $\mathrm{C} 1 \mathrm{q}$ in renal glomeruli of patients with proliferative lupus glomerulonephritis. Arthritis Rheum 1997; 40: 1504-1511

17. Cortes-Hernandez J, Fossati-Jimack L, Petry F et al. Restoration of $\mathrm{C} 1 \mathrm{q}$ levels by bone marrow transplantation attenuates autoimmune disease associated with $\mathrm{C} 1 \mathrm{q}$ deficiency in mice. Eur J Immunol 2004; 34: 3713-3722

18. Trouw LA, Groeneveld TWL, Seelen MA et al. Anti-C1q autoantibodies deposit in glomeruli but are only pathogenic in combination with glomerular C1q-containing immune complexes. J Clin Invest 2004; 114: 679-688

19. Trouw LA, Seelen MA, Duijs JMGJ et al. Glomerular deposition of $\mathrm{C} 1 \mathrm{q}$ and anti-C1q antibodies in mice following injection of antimouse C1q antibodies. Clin Exp Immunol 2003; 132: 32-39

20. Davis MM. A prescription for human immunology. Immunity 2008; 29: $835-838$

Received for publication: 12.1.10; Accepted in revised form: 19.8.10 